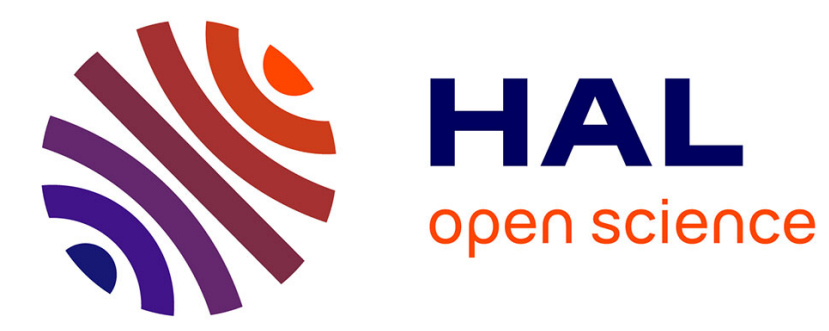

\title{
Géographie sociale et environnement
}

\author{
Monique Barrue-Pastor
}

\section{To cite this version:}

Monique Barrue-Pastor. Géographie sociale et environnement. Géographes associés, 1992, Sens et pratiques de l'environnement. Spécial Géoforum 91, 10, pp.58-62. 10.3406/geoas.1992.1767 . hal02865820

\section{HAL Id: hal-02865820 \\ https://hal.science/hal-02865820}

Submitted on 12 Jun 2020

HAL is a multi-disciplinary open access archive for the deposit and dissemination of scientific research documents, whether they are published or not. The documents may come from teaching and research institutions in France or abroad, or from public or private research centers.
L'archive ouverte pluridisciplinaire HAL, est destinée au dépôt et à la diffusion de documents scientifiques de niveau recherche, publiés ou non, émanant des établissements d'enseignement et de recherche français ou étrangers, des laboratoires publics ou privés. 


\section{Géographie sociale et environnement}

Monique Barrué-Pastor

\section{Citer ce document / Cite this document :}

Barrué-Pastor Monique. Géographie sociale et environnement. In: Géographes associés n¹0, 1er semestre 1992. Sens et pratiques de l'environnement. Spécial Géoforum 91 . pp. 58-62;

doi : https://doi.org/10.3406/geoas.1992.1767

https://www.persee.fr/doc/geoas_1266-4618_1992_num_10_1_1767

Fichier pdf généré le 09/11/2019 


\section{GÉOGRAPHIE SOCIALE ET ENVIRONNEMENT}

MONIQUE BARRUE-PASTOR

CIMA-URA 366du CNRS, TOULOUSE
La multiplicité des définitions données jusqu'ici à la notion d'environnement, atteste pour le moins de la difficulté à la cerner, sans doute à cause des questions de nature très diverse qui lui sont liée et des limites des approches sectorielles par "élément", qu'il s'agisse de l'eau, de l'atmosphère, des sols, de la faune ou de la flore.

Par ailleurs, nous ne pouvons plus nous contenter d'articulations simples entre "volontés humaines" et "possibilités naturelles", auxquelles nous ajouterions un peu de "territoire", un peu de "nature", et pour finir les conditions de travail et le cadre de vie. Les géographes semblent précisément avoir dans ce domaine beaucoup de choses à apporter mais aussi à dire puisque déjà de nombreuses études attestent à la fois de la spécificité et même d'une certaine originalité de leur apport, notamment par les géographes sociaux longtemps tenus à l'écart des "problématiques environnementalistes".

Si la notion d'environnement est une question socio-politique, il relève de notre responsabilité de chercheurs de ne pas laisser le discours globalisant sur l'environnement aux politiques et de contribuer à la construction d'un discours et d'une analyse scientifique.

\section{QUESTIONS PRÉALABLES}

Plusieurs remarques d'ordre général méritent d'être soulignées, en guise de préalable à l'expression des points de vue de géographes de spécialités diverses.

\subsection{Une prise de conscience faible et en retard}

Les géographes n'ont ni à se glorifier d'une certaine légitimité historique sur ces questions (..."la géographie ce n'est tout de même pas l'environnement"...) ni à se culpabiliser outre mesure sur le retard pris sur ce terrain, qui paradoxalement est certainement le plus proche de son paradigme central (rapports homme-société-nature). Ce retard s'inscrit dans un contexte national, où d'une manière générale la prise en considération de ces questions s'est faite de manière tardive (entre autre par rapport aux autres pays d'Europe).

\subsection{Les "problèmes environnementaux" sont en train de devenir une lame de fond...}

C'est-à-dire d'engendrer des changements significatifs. Ces changements concernent des questions essentielles : la conception du développement ; les rapports 
société-nature ; la conception des systèmes de gestion du territoire (qui introduit une nouvelle dimension en terme de régulation sociale des problèmes); la conception de la recherche.

Au-delà des multiples incertitudes qui demeurent, certaines nécessités apparaissent ; il s'agit bien de modifier :

- les problématiques : notamment par une prise en compte de la globalité et de la complexité;

- les pratiques interdisciplinaires, qui s'intensifient autour de la gestion des ressources naturelles, centrées sur la diversification des interactions entre dynamique des ressources et dynamiques des usages;

- les articulations entre différents niveaux d'échelle, en fonction de la nécessité d'explorer la multiplicité des interactions entre niveau local, région et niveau macro (national, international et mondial).

Les objectifs et les moyens peuvent se résumer en cinq mots-clés : valoriser les savoir-faire existants; re-orienter; re-articuler; recomposer; opérer un retour critique sur nous-mêmes pour dégager de nouvelles orientations de recherche.

L'enjeu est bien de jouer avec le caractère à la fois hétéroclite et fédérateur de la notion d'environnement (cf. travaux du groupe AGRER, dir. Marcel Jollivet). Hétéroclite, dans les outillages et les actes, mais aussi par la polysémie qui le caractérise et qui se retrouve dans l'outillage législatif réglementaire. Fédérateur, parce qu'il peut être un facteur d'intégration des politiques et des pratiques (jusqu'ici restées sectorielles), y compris de recherche.

\section{3. ...Pas d'entrée privilégiée ni} d'exclusive scientifique

L'opposition entre problématique à entrée spatiale et problématiques centrées sur les rapports entre société-nature semble pour le moins stérile. Les deux possibilités sont ouvertes à partir du moment où l'on considère que l'on peut sortir de l'espace pour poser les questions d'environnement et où certains processus ne sont pas spatialisés.

Si, dans une première phase c'est la dimension naturaliste qui a dominé les questions d'environnement... les choses évoluent vite...; actuellement, c'est la dimension sociale qui prime, notamment par l'articulation entre environnement et développement, et par la prise en compte de la dimension "cadre de vie".

Deux exemples de chantiers de recherche récents piochés dans ma pratique de géographe social, au CIMAURA 366 CNRS, permettront d'alimenter le débat de manière concrète.

\section{IMPACT DES MESURES SOCIO. STRUCTURELLES DE LA CEE : ÉVO. LUTION DES RAPPORTS ENTRE AGRICULTURE ET ENVIRONNEMENT}

Nous sommes en train de vivre un bouleversement tout à la fois récent et brutal des rapports entre agriculture et environnement, par double mécanisme : - l'intégration des préoccupations environnementales dans la P.A.C. (Politique Agricole Commune) entraîne une transformation des fonctions et des pratiques agricoles;

- l'intégration de l'agriculture dans les "pro- 
blèmes environnementaux" à la fois comme facteur de dégradation et de risque et à la fois comme moyen de protection et de lutte.

Nous ne sommes plus dans un système d'opposition de deux conceptions du rapport société/nature, mais dans une phase d'éclatement et de recomposition de la "nature", de "l'agriculture", et en conséquence de la "ruralité". Cette dernière nécessite d'être redéfinie en relation avec la notion d'environnement. Les rapports entretenus jusqu'ici entre agriculture et gestion $d u$ territoire sont modifiés. L'enjeu est bien de mettre en relation pour ne pas dire en complémentarité, deux objectifs conçus jusqu'ici comme contradictoires : celui de la protection et celui du développement.

C'est pourquoi nous avons envahi en tant que géographe (social), en relation avec d'autres secteurs de la géographie physique et avec d'autres disciplines (agronomie, écologie, économie...) un terrain réservé jusqu'ici (bien que très partiellement couvert) aux juristes et aux sociologues du droit. Il s'agit de l'analyse des législations nationales et européennes qui se multiplient depuis 1985 (depuis la première loi territorialisée : la loi Montagne qui fait figure de précurseur) et qui concernent l'agriculture et l'environnement. Au-delà de l'évaluation de leur contenu, c'est surtout la diversité de leurs conditions d'application et de leur impact territorial qui suscitent un intérêt renouvelé.

\subsection{L'arsenal législatif et réglementaire}

Il est constitué par l'ensemble des mesures socio-structurelles, en privilégiant les objectifs prioritaires contenus dans le 5A (accélérer l'adaptation des structures agricoles, notamment le "gel des terres", l'extensification et l'article 19) et le 5B ("promouvoir le développement des zones rurales"). L'inventaire critique de ces différents règlements permet de mettre en évidence les cohérences et les contradictions aux différents niveaux et échelles (GATT, PAC, France, Région, exploitation agricole) ; mais aussi certains paradoxes (trop et pas assez d'agriculteurs...).

L'intérêt essentiel résidant dans l'analyse des modifications significatives qu'ils engendrent dans :

- les fonctions de l'agriculture,

- la gestion des territoires (anciennement agricoles, notamment),

- la place et le statut des agriculteurs et des agricultures dans la société (au niveau économique, culturel, politique, idéologique), - la vision que les agriculteurs ont d'euxmêmes, comment ils intériorisent et vivent cette nouvelle situation, et par ailleurs, quel regard portent les autres catégories sociales sur les agriculteurs.

\subsection{Conditions d'application et diversité de leur impact territorial}

Le dernier point fort est, bien évidemment, l'impact spatial de ces règlements ; la diversité de leur mode d'application et de leurs effets selon les régions. Il y a modification des catégories d'espace, des types d'espace et des types de problèmes qu'ils posent.

Ces modifications peuvent véritablement changer le monde rural. Les géographes peuvent et doivent contribuer à cette analyse (au-delà de quelques choix individuels; leur apport y semble d'autant plus essentiel et même irremplaçable que ce 
champ de recherche concerne directement l'évolution des rapports entre nature et société. C'est du moins ce qu'attestent les résultats des recherches développées sur ce thème depuis plusieurs années au CIMA.

\section{RISQUES NATURELS, PAYSAN. NERIES ET AMÉNAGEMENTS MON. TAGNARDS}

L'investigation récente des sciences sociales dans l'approche des risques et des catastrophes a modifié autant les clés d'entrée que les problématiques et les résultats. Après une polarisation sur les cataclysmes, les séismes et les éruptions volcaniques qui ont stigmatisé une première phase de recherche très naturalistes, il y a eu un glissement par intégration de la dimension sociale, qui est en train de primer dans les recherches les plus récentes... Ici encore, peu de géographes occupent ce terrain, mais il en existe. Nous prendrons comme exemple les recherches effectuées par le CIMA' dans les Pyrénées, sur les risques et les catastrophes naturelles, parmi lesquelles le programme qui concerne le HautLavedan présente la plus large interdisciplinarité sous la responsabilité d'un géographe social ${ }^{2}$.

L'objectif est d'analyser dans une zone de montagne particulièrement touchée par les catastrophes et toujours soumise aux aléas non négligeables des risques "naturels", la réalité, les traces, les perceptions, les systèmes de représentation et les modes d'intégration de ces risques, tant dans les mémoires (individuelles et collectives), dans les pratiques et les modes de vie, dans l'architecture que dans les plans et projets d'aménagement.
Les catastrophes et les risques "naturels" sont donc analysés dans leur dimension physique, naturelle, sociale, psychologique. La multiplication des sources d'information, témoignages, enquêtes, descriptions, discours, recherchées autant dans les archives que sur le terrain aujourd'hui, rend possible une étude diachronique et synchronique, qui seule peut permettre d'éclairer la globalité et la complexité des phénomènes.

La multiplication des partenaires en est le corollaire : cette étude associe historiens, ethno-anthropologues, géographes physiciens et sociaux, linguistes, architectes et juristes. Le dispositif de recherche a été structuré autour de quatre points forts.

\subsection{L'importance de la dimension his- torique}

Elle concerne les rapports entre histoire des catastrophes, histoire des paysages et histoire de l'environnement.

Le dépouillement de toutes les sources d'archives disponibles permet de constituer une chronologie des catastrophes, une cartographie historique de leur localisation, de mesurer leur importance et d'évaluer leur répétitivité (travaux de S. Briffaud, B. Desailly, J.P. Métailié).

\subsection{Evaluation de l'Etat et de l'évolution des paysages}

L'objectif est d'apprécier l'inscription des risques dans le paysage (de 1860 à nos jours) ; compte tenu des aménagements effectués, d'évaluer la permanence des risques pour aboutir à une typologie qui 
intègre les notions de réversibilité et d'irréversibilité (travaux de J.P. Métailié, de B. Desailly et C. Carcenac).

\subsection{Les systèmes de représentations et pratiques d'aménagement}

L'analyse historique des discours sur la catastrophe et des pratiques d'aménagement (fin XVII" siècle-début XX" siècle : cf. travaux de $S$. Briffaud) est mise en correspondance avec la mémorisation, les systèmes de représentation et les pratiques locales des paysanneries montagnardes. La cartographie de cette "mémoire contemporaine" confrontée à la cartographie historique s'est avéré une source riche d'enseignements (cf. travaux de M. Barrué-Pastor). Elle facilite l'appréciation des modes d'intégration des risques dans les choix de gestion du territoire, dans les techniques de construction et les choix d'implantation des constructions, notamment des "granges foraines" (M. Barrué). L'apport des linguistes est ici irremplaçable pour décoder les dictons, les toponymes (en gascon), les rapports entre le "dire" et le "faire", mais aussi entre mythes, symboles et réalités (X. Ravier).

\subsection{Gestion des risques par les collectivités locales et procédures d'aménagement}

De la gestion des risques par les communautés montagnardes (XIX-XX" siècle, J.P. Allinne) aux Plans d'Exposition aux Risques (P.E.R.) : évolution de la prise en considération des rapports entre catastrophes et droit public (F. Ogé, R. Romi) mais aussi du degré de prise en compte des connaissances locales... entre paysans "marginalisés et ignorés" et aménageurs "scientistes", y a-t-il une amnésie de la logique du profit, dans ces vallées où le thermalisme et le tourisme ne cessent de se développer?

Ya-t-il changement dans l'évaluation économique du prix à payer contre le risque et aussi dans la relativité et la diversité du "coût" selon les catégories sociales?

Même si de nombreux éléments d'interrogation demeurent, qui constituent l'intérêt des recherches de demain, ces quelques éléments de réponse prouvent la capacité des géographes à dynamiser des équipes interdisciplinaires et à intervenir à différents échelons des problèmos d'environnement.

\section{NOTES}

1- Bertrand DESAILLY : "Crues et inondations en Roussillon. Le risque et l'aménagement. Fin du XVIle siècle-milicu du XX" siècle", Thèse de Doctorat de Géographie, Nanterre, 1990, 341 pages.

Collectif : "Le torrent et le fleuve : risques, catastrophes et aménagement dans les Pyrénées et leur piémont. Fin du XVII" siècle-XX" siècle", Rapport de recherche PIREN : Histoire de l'environnement, Toulouse, février 1991, 313 pages (sous la responsabilité de Jean-Paul Métailié).

2- Collectif : "De la mythologie des catastrophes à leur gestion. Analyse interdisciplinaire d'un cas exemplaire : la vallée des Barèges. XVII ${ }^{\circ}$-XX" siècles", Rapport de fin de recherche, Ministère de l'environnement, Toulouse, juillet 1991 (sous la responsabilité de Monique Barrué-Pastor) 